\title{
BIOMETRIC BASED STUDENTS' BUS MANAGEMENT SYSTEM
}

\section{R. BHUVANYA \& S. UMA}

Assistant Professor, Department of CSE \& VELTECH DR RR \& DR SR UNIVERSITY, Chennai Tamil Nadu, India

ABSTRACT
This paper aims at designing a student monitoring system, which could effectively manage presence of students
at school buses. Student's presence is marked after each student's identification. For the identification of student, a
fingerprint recognition system is used. Fingerprints are considered to be the best and fastest method for biometric
identification, since it is very secure to use and that cannot be changed in one's life time. So, the framework is developed
for managing the students' attendance based on fingerprint. Fingerprint reader is placed in the school bus, hence
whenever the student boards into the bus, the students have to place their biometric to indicate the presence of arrival and
departure. This biometric takes attendance electronically, and the records of the attendance are sent digitally to the school
database. The database in turn generates a text message and sends it to the corresponding parents indicating the safer
departure and arrival time of their children.
KEYWORDS: Monitoring System, Biometric Takes \& Arrival and Departure

Received: Nov 12, 2017; Accepted: Dec 03, 2017; Published: Jan 05, 2018; Paper Id.: IJMPERDFEB201819

\section{INTRODUCTION}

In schools and organizations, we are giving much importance to the attendance during start and end of the day to mark a person's presence. Traditional ways of taking attendance is, like recorded manually in attendance register by calling out names, but this result in waste of time and human effort. And also, there is a possibility of errors that may happen while the people work with the register. And, these issues will be high when the class strength is more. A solution to overcome this problem is by using the system that will record the student's attendance, based on their biometric recognition. In this direction, this paper suggests the method to record the student attendance automatically. This system consists of a fingerprint sensor in the school bus that helps for identifying the student. Here, the student needs to place their finger on the fingerprint sensor to obtain their attendance. This proposed method not only helps the educational institutions to mark the presence of students, but also help the parents to inform about the safest arrival of their children in the morning. While in evening, if the student finger print is recognized, then the parents will get a text message about the departure time of their children. Hence, the usage of this system will reduce the parent's tension towards their children.

Biometric identification systems are in wide use for the unique identification of humans, especially for the purpose of verification and identification. Biometrics is used as a form of access control and identity management. Hence, this biometrics in student's attendance management system is a secure approach. Though we have many types of biometric systems like fingerprint recognition, face recognition, voice recognition, iris and ear recognition systems. In this paper, the focus is about fingerprint recognition system. This biometric system is further classified into unimodal and multimodal. Face and finger print recognition falls under the category of unimodal, and if the person tries to get multiple information from more than one biometric modality that comes 
under multi modal.[1]

Our skin has some patterns of ridges and valleys. Papillary edges on the finger is also called as friction edges, which helps to grasp objects and also increase the friction and improve the tactile sensing. And all that we know is, ridge patterns are scientifically proved as unique for each person. If there is any cuts and burns in a person's finger, that may alter the patterns, but it is said to be a temporary one. Finger prints are now used widely in identification and verification purposes. They are used for attendance purpose in some institutions which avoid the proxy and illegal entries. Here in this paper, we have tried to automate an attendance procedure for the students regarding their safer departure and arrival. This automated system can be used for verification, identification and indication, since it is done at the school bus itself. Here, the system compares an input finger with the enrolled finger print of the particular user to determine, if they are from the same finger, whereas, in the identification stage, the system compares an input finger print with the print of all enrolled users in the database to check their identity.

Biometric technologies verify the characteristics of fingerprints, faces, voice, hand written signature and so on. It uses the physical data which receives the attention as a personal authentication method, which is more convenient than other conventional methods such as a retrieval of password or ID cards, since the data has been taken from measurements and such data is unique to every individual, and it remains so throughout one's life time. The importance of attendance management system is described as below.

In a human resource management system, attendance management is a major part. Hence, punctuality and regular attendance are expected from all students in the institutions. Purpose of the attendance is very important, since it will be used for the record keeping, assessment of students and that will act as an indirect loop hole of knowledge acquiring. In a traditional method, the uses of sheets of paper or books are used in taking attendance presents. But, there is a possibility for impersonation and the attendance sheet could be stolen or lost. Taking attendance is a time consuming process, and also it is difficult to ascertain the parents about the safety departure and arrival time of a student. Thus, the system is designed to mark the presence of students with their earliest and safest reach ability. This is the reason why the finger print reader is placed in the school bus itself.

\section{Advantages of Biometric Include}

\section{Cannot be Forgotten or Lost}

Tokens such as papers can be lost and magnetic strips such as RFID can be lost or stolen even it can be duplicated i.e. there is a chance of impersonation. But on the other hand, biometric verification needs the physical presence of the user and it will never get forged due to the unique ridge patterns.

\section{Improved Security}

In biometric verification system, users can not predict multiple identities and thus it helps to screen the users. And also, it requires minimum training to get the system operational and it will never expensive password administrators.

\section{Environment Friendly}

It reduces paper and other resource which in turn do not cause any negative impact to the environment. Here, the software and hardware can be easily used with 


\section{Time Saving}

It is extremely quick to identify the person in a micro second. The business people, who are aware of time management, will use this technology thus leads to increased productivity and reducing costs which eliminates the fraud and paper waste technology.

\section{RELATED WORK}

- In this project, author presents the biometric identification system to recognize the student attendance based on the ratio of the false acceptance rate (FAR) and false reject rate (FRR). Here, they followed two stages for marking the student attendance. Initially in the enrollment stage, biometrics of the student is recorded and the ridge patterns are uniquely identified and stored in the database. At the later stage of authentication, the extracted features are compared with the existing data base and the ratio will be calculated for FRR Vs FAR.

- In the attendance management, it is done using the handheld finger print sensor. Here, the enrolment module focuses on capturing the bio metrics, and it is stored in a flash memory with the person's id. The recognition will be done in the authentication module by comparing the finger print with the one, that is stored in the flash memory, and if the id matches then the student attendance is marked. Usage of flash memory improvises the access time. Since the flash memory is integrated with the micro controller, its usage became easier.

- Focuses on the wireless attendance management system using the ZigBee technology. It can be achieved by employing the finger print module and GPS system. Zig Bee chip is integrated with micro controller and operates in ISM radio frequency bands. In this framework, the login form is presented to the students. Students will be given the username and password. After entering the login credentials, students will select the particular course with the appropriate semester. Once the details are submitted by the student, then the attendance will gets updated.

- Emphasis the work on finger print based attendance management system with the minutiae extraction. In the enrolment process, biometric image is captured and the preprocessing of minutiae extraction is done. After the extraction, the particular image will be stored in the database. And, that forms the basis for authentication. Whereas, at the authentication process, the features of the user is compared with the existing template to determine the particular match. At the later stage, if the match is found, then the attendance will be marked.

- Implemented an automatic attendance system based on a real time Computer vision algorithm (CV). It integrates a face vision algorithm and computer vision to mark the attendance of student. Though it eliminates the traditional method of calling out names, the drawback behind is, there is a possibility in facial image that may gets changed between the time of enrolment and authentication. Hence, it provides a lower recognition rate, somehow at the extent; it deals with a financial burden and does not include any protection for the privacy.

- Implementation of automated attendance management system with the android device is the work focused on [6]. The students have to enter their login credentials and they have to enter their personal details like (name, course, semester etc). Similarly, the teacher is also assigned with the username and password. This will be maintained in a separate database. Every day, the teacher has to activate the application on the server. If it is activated, then the student can mark their attendance with a single click. If the teacher wants to see the report of the student, then the facility is included in the application. If he/she wants to generate the monthly report, then can be done through this 
application and the report will be delivered to the parents.

\section{SYSTEMDESIGN}

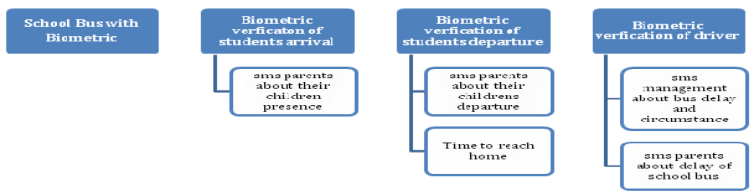

Figure 1: System Design

\section{IMPLEMENTATION}

Fingerprint based attendance management system has the following modules

- Student's Biometric module

- Recognition Module

- $\quad$ System database

- Transmission of text message

\section{Students Biometric Module}

The task of enrollment module is to enroll the student and their biometrics into the school database. At this stage, finger biometric image will be captured for every student. When the image is captured, at the enrolment stage two image samples are taken for acquiring high level accuracy. For each biometric image, unique features are extracted by applying minutiae extraction algorithm. Separate ID is assigned to each student with the extracted feature. This ID comprises of student name, Class, Section, passport photograph, Parent's phone number. The extracted images form a pattern which helps to identify the particular student. Here, all the process is carried out by the administrator.

Each detected minutiae is characterized by four parameters. Typically it is said to be,

$\mathrm{m}_{\mathrm{i}}=\left(\mathrm{x}_{\mathrm{i}}, \mathrm{y}_{\mathrm{i}}, \Theta_{\mathrm{i}}, \mathrm{t}_{\mathrm{i}}\right) \quad$ where:

$\mathrm{x}_{\mathrm{i}}, \mathrm{y}_{\mathrm{i}}-$ are coordinates of the minutiae point,

$\Theta_{i}-$ is minutiae direction typically obtained from local ridge orientation,

$t_{i}$ - is type of the minutiae point (ridge ending or ridge bifurcation. The matching phase of the algorithm performs two functions.

There are a lot of minutiae extraction techniques available. They are predominantly classified into,

- Direct level minutiae extraction

- Binary image - based minutiae extraction

- Machine learning method and

- Skeletonization -based minutiae extraction.

Out of these, this Binary image based minutiae extraction can be chosen which is further classified into, unthinned 
binarized image and Thinned binarized image. Here, the Morphology based technique is applied and it is based on the thinned binarized image. The procedure for morphology based technique is as follows: At the initial stage, the image is preprocessed to reduce the efforts needed in post processing stage. Preprocessing operations will remove the spurs, bridges etc then the true minutiae extracted based on the miss transform and morphological hit techniques. The morphological operators allow the manipulation of shapes, which helps for the identification of each biometric. The structuring elements can be developed for different types of minutiae, which are then used by the Hit or Miss transform to extract the valid minutiae for each biometric.

\section{Authentication Module}

The authentication module works as follows: It tries to validate the identity of the student based on the pattern extracted with the minutiae extraction algorithm. The extracted patterns are fed into the matching algorithm which matches the pattern of the student stored in the school database. After the successful match, the attendance will be marked against the particular student, and the text message will be sent to the parent's phone number regarding the safer arrival and departing time of their children. Though the finger print matching involves the approaches of minutiae based matching, ridge based matching and correlation matching procedures, here we apply the minutiae based extraction, which facilitates the design of robust and fast verification algorithm and storage efficient. Matching algorithm splits the task by,

(1) Separates the Candidate Common Points List into two lists,

○ Confirmed Common Points List and

- Spurious / Unconfirmed Point List.

(2) Uses the Confirmed Common Points List to generate a Matching Score between the Base and the Input image.

Performance of the identification system can be measured based on the FPIR and FNIR. FPIR is a false positive identification rate that occurs, when the system tries to find a fingerprint that is not enrolled in the school database. On the other hand, FNIR is a false negative identification rate occurs, when the system fails to find a particular ID that is enrolled in the database. Hence, the relationship between the rates can be defined as, FPIR = $\mathbf{1}-(\mathbf{1}-\mathbf{F M R}) \mathbf{N}$, where N denotes the no of students list stored in the database.

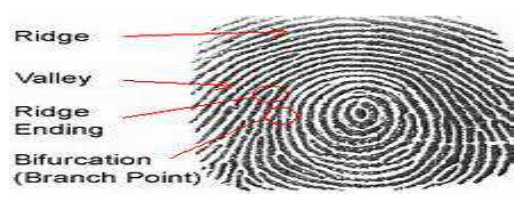

Figure2: Sample Biometric image

\section{System Database}

School database is created and maintained by administrator. Here, the database comprises of Student's name, student ID, Class, Section, Passport Photograph, parents phone number, address. Whenever the student biometric is recognized, the parents will receive the text message of safer arrival/ departure of their children. The transmission of text message can be done by retrieving the parents phone number from the database stored. The database is designed with the structured query language (SQL). With this, we can retrieve the large amount of records from a database quickly and efficiently. It is the database language, which is used widely in business and enterprise applications throughout the globe. Specifically to work with the enterprise applications, it is a perfect language for database. Previously, SQL databases were 
synonymous with the relational database. Now, with the evolution of OODBMS, object storage capabilities are extended to relational database.

\section{Transmission of Text Message}

This elaborately explains the transmission of text message to an appropriate phone number stored in the database. Here, every student's biometric is mapped with their parent's phone number. When the biometric is matched, then the particular phone number will be identified through the school database and the text message will be forwarded to the parents. With this, the parents can feel secured about their children and they can calculate the arrival time to their home, when the departure time of their children is sent. And also, the parents can feel relaxed hence they can get to know about their children in the morning and in the evening. So, there is no way of missing the child. And, if any particular student forgets to place the biometric in the school bus at evening time, then the parents can ask the concerned class coordinator and they can get to know about the special class conducted. While the time of travel, if any issue occurs in the school vehicle, then the driver can place his biometric with the device installed in the school bus, to indicate the fault occurred to the school management. As soon as the driver's biometric is identified, then the supervisor can immediately arrange for the alternative vehicle, and the respective message will be transmitted to the parents. If the parents are aware of the school vehicle, then the management will not give a chance for fault occurrence.

\section{CONCLUSION AND FUTURE SCOPE}

With the secure schooling using biometric, we can conclude that the students will reach their destination safely. Since the parents are aware of the departure and arrival timings, no one can get away from their respective destination. And also, the management will try to eliminate even a single chance of faulty occurrence, since they are being monitored by their stack holders directly. This system can be extended to colleges and universities also.

\section{REFERENCES}

1. O. Shoewul, N.T. Makanjuola, S.O. Olatinwo “Biometric-based Attendance System: LASU Epe Campus as Case Study”, International American Journal of Computing Research Repository, 2014, Vol. 2, No. 1, 8-14

2. Karthik Krishnamurthi, S. Irudaya Mary, B. N. Sumalatha, Adler Pereira "Fingerprint Based Attendance System" International Journal of Advanced Research in Computer and Communication Engineering, Vol. 4, Issue 3, March 2015

3. A. D. Bhople, A New Approach for Person Verification System Based on Finger-Knuckle Print Biometric and MLP Neural Network, International Journal of Computer Science Engineering and Information Technology Research (IJCSEITR), Volume 5, Issue 2, March - April 2015, pp. 79-88

4. Sanjay Badhe, Kunal Chaudhari, Sneha Kale, Tejaswi Mane “Smart Attendance Management System”, International Journal of Computer Applications, National Conference on Advancements in Computer \& Information Technology (NCACIT-2016)

5. Akinduyite C.O, Adetunmbi A.O, Olabode O.O, Ibidunmoye E.O "Fingerprint-Based Attendance Management System", Journal of Computer Sciences and Applications, 2013, Vol. 1, No. 5, 100-105

6. Visar Shehu, Agni Dika "Using Real Time Computer Vision Algorithms in Automatic Attendance Management Systems", Proceedings of the ITI 2010 32nd Int. Conf. on Information Technology Interfaces, June 21-24, 2010, Cavtat, Croatia

7. Aniket Shah, Amruta Tuptewar, Pradnya Yeole, Prof.L.J.Sankpal "Automated Attendance Monitoring and Personal Intelligent system”, International Journal of Advanced Research in Computer and Communication Engineering Vol. 3, Issue 11, November 2014 